\title{
Risk of tumor recurrence in intracranial meningiomas: comparative analyses of the predictive value of the postoperative tumor volume and the Simpson classification
}

\author{
*Dorothee Cäcilia Spille, MD, ${ }^{1}$ Katharina Hess, MD, ${ }^{2}$ Eike Bormann, MSc, ${ }^{3}$ \\ Cristina Sauerland, MSc, ${ }^{3}$ Caroline Brokinkel, MD, ${ }^{4}$ Nils Warneke, MD, ${ }^{1}$ Christian Mawrin, MD, ${ }^{5}$ \\ Werner Paulus, MD, ${ }^{2}$ Walter Stummer, MD, ${ }^{1}$ and Benjamin Brokinkel, MD ${ }^{1}$

\begin{abstract}
${ }^{1}$ Department of Neurosurgery, ${ }^{2}$ Institute for Neuropathology, and ${ }^{4}$ Institute for Clinical Radiology, University Hospital Münster; ${ }^{3}$ Institute of Biostatistics and Clinical Research, University of Münster; and ${ }^{5}$ Institute of Neuropathology, Otto von Guericke University Magdeburg, Saxony-Anhalt, Germany
\end{abstract}

\begin{abstract}
OBJECTIVE In meningiomas, the Simpson grading system is applied to estimate the risk of postoperative recurrence, but might suffer from bias and limited overview of the resection cavity. In contrast, the value of the postoperative tumor volume as an objective predictor of recurrence is largely unexplored. The objective of this study was to compare the predictive value of residual tumor volume with the intraoperatively assessed extent of resection (EOR).

METHODS The Simpson grade was determined in 939 patients after surgery for initially diagnosed intracranial meningioma. Tumor volume was measured on initial postoperative MRI within 6 months after surgery. Correlation between both variables and recurrence was compared using a tree-structured Cox regression model.
\end{abstract}

RESULTS Recurrence correlated with Simpson grading ( $p=0.003)$. In 423 patients $(45 \%)$ with available imaging, residual tumor volume covered a broad range $\left(0-78.5 \mathrm{~cm}^{3}\right)$. MRI revealed tumor remnants in $8 \%$ after gross-total resection (Simpson grade I-III, range $0.12-33.5 \mathrm{~cm}^{3}$ ) with a Cohen's kappa coefficient of 0.7153 . Postoperative tumor volume was correlated with recurrence in univariate analysis (HR 1.05 per $\left.\mathrm{cm}^{3}, 95 \% \mathrm{Cl} 1.02-1.08 \mathrm{per} \mathrm{cm}^{3}, \mathrm{p}<0.001\right)$. A tree-structured Cox regression model revealed any postoperative tumor volume $>0 \mathrm{~cm}^{3}$ as a critical cutoff value for the prediction of relapse. Multivariate analysis confirmed the postoperative tumor volume (HR 1.05, $p<0.001)$ but not the Simpson grading ( $p=0.398)$ as a predictor for recurrence.

CONCLUSIONS EOR according to Simpson grading was overrated in $8 \%$ of tumors compared to postoperative imaging. Because the predictive value of postoperative imaging is superior to the Simpson grade, any residual tumor should be carefully considered during postoperative care of meningioma patients.

https://thejns.org/doi/abs/10.3171/2020.4.JNS20412

KEYWORDS meningioma; microsurgery; recurrence; Simpson grade; volumetry; oncology

$\mathrm{R}$ ESECTION remains the treatment of choice in symptomatic and/or space occupying intracranial meningiomas. ${ }^{1}$ In 1957, Donald Simpson proposed a simple method for quantifying the extent of resection (EOR) according to the neurosurgeons' intraoperative assessment and, accordingly, to estimate the risk of postoperative tumor recurrence. ${ }^{2}$ Nowadays, the Simpson grading system has been widely established to quantify the EOR in clinical routine and research, including current prospective trials. ${ }^{3,4}$ However, the prognostic value of the
Simpson classification system is increasingly discussed, ${ }^{5-18}$ and more recent series have questioned its uniform applicability to tumors in different intracranial locations and to recurrent lesions. ${ }^{19,20}$ In addition, derived dichotomous scales distinguishing gross-total resection (GTR) and subtotal resection (STR) are widely used to quantify the extent of tumor removal in retrospective studies, but also in currently ongoing clinical trials. ${ }^{3,11,13,14,19,20}$ It is noteworthy that definitions of both dichotomizations, particularly with regard to the classification of Simpson grade III resections,

ABBREVIATIONS EOR = extent of resection; GTR = gross-total resection; KPS = Karnofsky Performance Scale; PFI = progression-free interval; STR = subtotal resection. SUBMITTED February 10, 2020. ACCEPTED April 21, 2020.

INCLUDE WHEN CITING Published online July 17, 2020; DOI: 10.3171/2020.4.JNS20412.

${ }^{*}$ D.C.S., K.H., and B.B. contributed equally to this work. 
remain controversial, and conflicting descriptions are even found comparing the pioneering work of Simpson with current meningioma treatment guidelines. ${ }^{1,2}$

Along with the increased discussion about the Simpson classification system and the heterogeneous definitions of GTR and STR, the question arises as to whether subjective intraoperative assessment of the EOR is equivalent to objective evaluation on postoperative MRI. ${ }^{21-23}$ Recent studies have shown the feasibility of automatic computerized segmentation in meningiomas and other CNS neoplasms, allowing precise tumor volumetry and further emphasizing the importance of differentiated imaging analysis. ${ }^{24-26}$ However, correlations between the postoperative tumor volume and the risk of recurrence in meningiomas are sparsely investigated and usually restricted to small series and distinct tumor locations. ${ }^{21-23}$

Therefore, in this study, we compared the prognostic value of the Simpson grading system-derived dichotomous classifications and the imaging-derived postoperative tumor volume for prediction of recurrence in a large series of patients undergoing operations for intracranial meningioma. Moreover, we elucidate differences between the intraoperatively assessed EOR and the residual tumor volume on postoperative imaging.

\section{Methods}

\section{Patients and Data Collection}

Medical and operative reports from all patients who underwent microsurgery for intracranial meningiomas in the University Hospital Münster Department of Neurosurgery between 1991 and 2018 were reviewed according to previous descriptions..$^{19,20,27-31}$ Collected data included the following: patients' sex and age at the time of surgery; preoperative Karnofsky Performance Scale (KPS) score; ${ }^{32}$ indication for surgery (primary or recurrent meningioma); tumor location, classified as "skull base" and "non-skull base" lesions, the latter including convexity and falcine/ parasagittal meningiomas and tumors arising from other intracranial non-skull base locations; administration of adjuvant radiation; and the grade of resection according to the Simpson classification system, assessed intraoperatively by the neurosurgeon. As originally stated by Simpson, we referred to Simpson grade I as a complete resection with excision of the dura attachment; Simpson grade II as resection of all visible tumor remnants and coagulation of the dura attachment; Simpson grade III as a macroscopically complete resection without coagulation of the dura attachment and with the possibility of remaining tumor in the venous sinus or by en plaque meningioma; Simpson grade IV as incomplete resection; and Simpson grade $\mathrm{V}$ as biopsy. For further analyses, the EOR was classified according to the two most commonly published definitions of GTR and STR (Simpson grade I-II vs $\geq$ III, and Simpson grade I-III vs $\geq$ IV). Histopathological diagnosis and grading had been established according to the current 2016 WHO criteria in all cases. ${ }^{33}$

Initial routine postoperative gadolinium-enhanced MRI was generally scheduled at 3 months after surgery. For this study, only MRI performed within 6 months after surgery was considered to reduce the probability of de- tecting early postoperative progression instead of residual tumor tissue. Imaging was analyzed by a team of two independent observers (D.C.S. and C.B.) without access to the operative reports. Residual tumor was identified on T1-weighted axial, coronal, and sagittal imaging, and if necessary, differentiated from local postoperative changes (e.g., duraplasty) taking into consideration preoperative imaging and operative reports. Volumetry was performed using commercial neuronavigation software (Brainlab version 2.6 neuronavigation system, Brainlab AG).

Follow-up imaging was repeated every year and every 6 months in benign and high-grade meningiomas, respectively. ${ }^{1}$ After an event-free follow-up of 5 years, imaging was repeated after 24 and 12 months in Simpson grade I and II/III tumors, respectively. In patients with contraindications for MRI, contrast-enhanced CT scans were performed for surveillance. Imaging was analyzed for recurrence of totally resected or progression of subtotally removed lesions by a team of at least 1 neurosurgeon and 1 neuroradiologist. Data about progression were additionally updated using standardized questionnaires, which were sent to the primary caretakers. Progression-free interval (PFI) was defined as the duration between index surgery and radiologically confirmed tumor recurrence, or in cases of an event-free follow-up, to the date of last follow-up.

\section{Statistical Analyses}

All calculations were performed using statistical software (IBM SPSS Statistics, version 25, IBM Corp.; R version 3.6.0, the R Foundation for Statistical Computing; and SAS version 9.4, SAS Institute) and data were characterized by standard statistics. Hence, continuous variables are described by median and range and compared using the Mann-Whitney U-test, and categorical variables are described by absolute and relative frequencies and compared using Fisher's exact test. Additionally, odds ratios (ORs) were computed to further examine the relationship between Simpson grade (I-III vs IV/V) and tumor location. PFIs were analyzed by the Kaplan-Meier method and compared using log-rank tests. PFI was further investigated by multivariate analyses using Cox regression, including the following variables: patients' age and sex, tumor location, histology, postoperative tumor volume $\left(\mathrm{cm}^{3}\right)$, and the intraoperatively assessed EOR, according to descriptions in the corresponding sections (see reference groups in Tables 1 and 2). The results are characterized by hazard ratios (HRs), 95\% confidence intervals (CIs), and Wald test $\mathrm{p}$ values. Reliability of the intraoperative assessment of GTR (Simpson grade I-III) was compared with an expected volume of $0-\mathrm{cm}^{3}$ tumor remnants on postoperative imaging and characterized by Cohen's Kappa statistic. Regression tree models with Simpson grade and residual tumor volume (step width $0.5 \mathrm{~cm}^{3}$ ) as independent covariates were used to find the best partition for prediction of PFI. Five hundred iterations were performed, during which the data set was randomly split into a training set and a validation set. Final model selection was based on the C-index on the validation set. All reported $\mathrm{p}$ values are 2 -sided and considered statistically significant when $<0.05$. Data collection and scientific use were approved by the local ethics committee. Patient consent was required in each case. 
TABLE 1. Clinical and histological risk factors for tumor recurrence in univariate analyses $(n=939)$

\begin{tabular}{|c|c|c|c|}
\hline Variable & $\mathrm{HR}$ & $95 \% \mathrm{Cl}$ & $\mathrm{p}$ Value \\
\hline Age & 1.07 & $0.99-1.02$ & 0.368 \\
\hline \multicolumn{4}{|l|}{ Sex } \\
\hline Female & Ref & Ref & \\
\hline Male & 2.24 & $1.54-3.24$ & $<0.001$ \\
\hline \multicolumn{4}{|l|}{ Tumor location } \\
\hline Non-skull base & Ref & Ref & \\
\hline Skull base & 1.31 & $0.90-1.89$ & 0.158 \\
\hline \multicolumn{4}{|l|}{ WHO grade } \\
\hline I & Ref & Ref & \\
\hline$\|/ /\|$ & 4.44 & $3.03-6.50$ & $<0.001$ \\
\hline \multicolumn{4}{|l|}{ Simpson grade } \\
\hline 1 & Ref & Ref & \\
\hline II & 1.727 & $1.04-2.87$ & 0.035 \\
\hline III & 1.85 & $0.99-3.43$ & 0.053 \\
\hline IV/V & 3.30 & $1.79-6.08$ & $<0.001$ \\
\hline Simpson grade $1 / I I$ & Ref & Ref & \\
\hline Simpson grade III-V & 1.68 & $1.14-2.48$ & 0.008 \\
\hline Simpson grade I-III & Ref & Ref & \\
\hline Simpson grade IV/V & 2.20 & $1.36-3.56$ & 0.001 \\
\hline
\end{tabular}

Male sex, high-grade histology, and intraoperatively assessed EOR were correlated with recurrence.

\section{Results}

Altogether 1306 patients underwent surgery for meningioma in our institution between 1991 and 2018. Figure 1 illustrates patient selection for subsequent statistical analyses. Nine hundred thirty-nine patients were included, including 671 females (71\%) and 268 males (29\%), with a median age of 58 years (range 7-91 years), primary diagnosed intracranial meningioma, and available information about the EOR. Table 3 summarizes the baseline clinical and histopathological data. With a median follow-up of 37 months, tumor recurrence was observed in 112 cases (12\%).

\section{Correlation Between Simpson Grading and Risk of Tumor Recurrence}

Table 1 summarizes risk factors associated with tumor recurrence in univariate analyses. Recurrence was observed in 21 (8\%), 51 (11\%), 19 (18\%), 20 (19\%), and $1(25 \%)$ patients after Simpson grade I, II, III, IV, and V resections, respectively $(\mathrm{p}=0.003)$. Correspondingly, PFI significantly decreased with increasing Simpson grade $(\mathrm{p}$ $=0.003$, Fig. 2). The risk of STR (Simpson grade $\geq$ IV) was distinctly higher in skull base than in non-skull base lesions (OR 6.02, 95\% CI 3.70-9.80; p < 0.001).

\section{Differences in the Prognostic Value of Established Dichotomizations of the EOR}

Seventy-two (10\%) of 726 patients after Simpson grade I and II resections developed progression, whereas 40 (19\%) of 213 patients developed progression after Simpson grade III-V resections $(\mathrm{p}=0.001)$. PFI was signifi-
TABLE 2. Multivariate analyses of risk factors for tumor recurrence in 423 patients with available postoperative MRI performed within 6 months after surgery

\begin{tabular}{|c|c|c|c|}
\hline Variable & HR & $95 \% \mathrm{Cl}$ & p Value \\
\hline Age & 1.00 & $0.98-1.02$ & 0.967 \\
\hline \multicolumn{4}{|l|}{ Sex } \\
\hline Female & Ref & Ref & \\
\hline Male & 1.756 & $0.909-3.393$ & 0.0939 \\
\hline \multicolumn{4}{|l|}{ Tumor location } \\
\hline Non-skull base & Ref & Ref & \\
\hline Skull base & 1.016 & $0.510-1.947$ & 0.9621 \\
\hline \multicolumn{4}{|l|}{ WHO grade } \\
\hline I & Ref & Ref & \\
\hline$\|/\| \|$ & 3.401 & $1.784-6.485$ & 0.0002 \\
\hline \multicolumn{4}{|l|}{ Simpson grade } \\
\hline I & Ref & Ref & 0.3981 \\
\hline$\|$ & 2.773 & $0.815-9.443$ & 0.1027 \\
\hline III & 3.287 & $0.750-14.409$ & 0.1145 \\
\hline IV/V & 2.592 & $0.613-10.955$ & 0.1952 \\
\hline Postop tumor volume & 1.05 & $1.02-1.08$ & 0.0007 \\
\hline
\end{tabular}

High-grade histology and the volume of residual tumor tissue, but not the EOR according to the Simpson grade, were shown to be strong risk factors for recurrence.

cantly longer after Simpson grade I and II resections than after Simpson grade $\geq$ III resections $(p=0.008$, median PFI not reached after median follow-up). Similarly, $11 \%$ of patients developed recurrence after Simpson grade I-III resections $(\mathrm{n}=91)$, but $19 \%$ of patients developed recurrence after Simpson grade IV or V resections $(\mathrm{n}=$ $21, p=0.018)$, and PFI significantly differed between both groups $(\mathrm{p}=0.001$, median PFI not reached after median follow-up).

\section{Postoperative Imaging, Postoperative Tumor Remnants, and Simpson Grade I-V Resections}

Early postoperative imaging eligible for volumetric analyses was available in 423 patients (45\%) and was performed after a median of 2 months (range 0-6 months) after surgery. The median postoperative tumor volume was $0 \mathrm{~cm}^{3}$ (range $0-78.5 \mathrm{~cm}^{3}$ ). Table 4 summarizes the postoperative tumor volumes after different Simpson grades. The median tumor volume was $0 \mathrm{~cm}^{3}$ after Simpson grade I, II, or III resections. However, residual tumor tissue was detectable after Simpson grade I resections in $5(5 \%)$ of 95 cases (range $0.56-4.07 \mathrm{~cm}^{3}$ ), after Simpson grade II surgeries in $17(7 \%)$ of 235 cases (range $0.12-33.5$ $\left.\mathrm{cm}^{3}\right)$, and after Simpson grade III resections in $6(23 \%)$ of 26 cases (range $0.27-9.55 \mathrm{~cm}^{3}$ ). Similar to the Simpson grades, postoperative tumor volumes were higher in skull base than in non-skull base lesions (median $0 \mathrm{~cm}^{3}$, range $0-78.50 \mathrm{~cm}^{3}$, vs $0 \mathrm{~cm}^{3}$, range $0-33.50 \mathrm{~cm}^{3}$; $\left.\mathrm{p}<0.001\right)$. As expected, the median residual tumor volume $\left(4.79 \mathrm{~cm}^{3}\right)$ was larger and varied widely after Simpson grade IV or V surgeries (range $0.00-78.5 \mathrm{~cm}^{3}$ ). Cohen's Kappa statistic between intraoperative assessment of GTR (Simpson grade I-III) and residual tumor volume $\left(0 \mathrm{~cm}^{3}\right)$ was 0.7153 


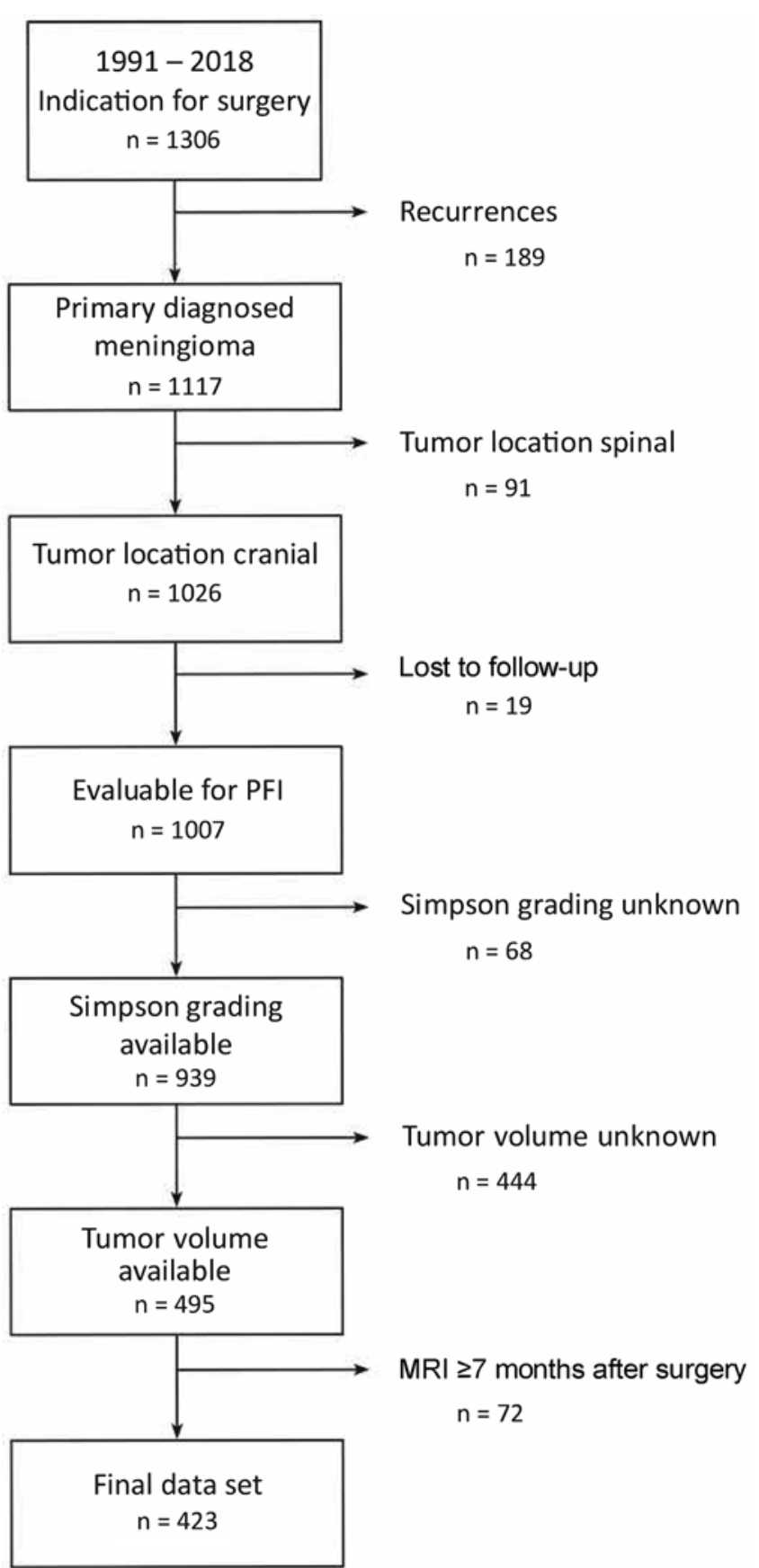

FIG. 1. Flowchart of patient selection.

(95\% CI 0.6286-0.8020). The cases intraoperatively misclassified as GTR were mainly located in the skull base ( $\mathrm{n}=18 / 28,64 \%$ ). However, after omitting one case with relevant residual tumor volume of $33.50 \mathrm{~cm}^{3}$, the median postoperative tumor volume of this subgroup was $2.75 \mathrm{~cm}^{3}$ (range $0.12-9.55 \mathrm{~cm}^{3}$ ).

Among 423 patients with available postoperative MRI, recurrence was observed in 46 cases $(11 \%)$ and was positively correlated with residual tumor volume (HR 1.04 per $\mathrm{cm}^{3}, 95 \%$ CI 1.02-1.06 per $\mathrm{cm}^{3}, \mathrm{p}<0.001$; Fig. 3). In multivariate analyses adjusted for age, sex, tumor loca-
TABLE 3. Baseline clinical and histopathological characteristics $(n=939)$

\begin{tabular}{|c|c|c|}
\hline Variable & Value & $\%$ \\
\hline Median age (range), yrs & $58(7-91)$ & \\
\hline \multicolumn{3}{|l|}{ Sex } \\
\hline Male & 268 & 28.5 \\
\hline Female & 671 & 71.5 \\
\hline \multicolumn{3}{|l|}{ Tumor location } \\
\hline Non-skull base & 520 & 55.4 \\
\hline Skull base & 419 & 44.6 \\
\hline \multicolumn{3}{|l|}{ KPS score } \\
\hline$\geq 80$ & 805 & 86.0 \\
\hline$<80$ & 131 & 14.0 \\
\hline \multicolumn{3}{|l|}{ WHO grade } \\
\hline I & 825 & 87.9 \\
\hline$\|/ /\|$ & 114 & 12.1 \\
\hline \multicolumn{3}{|l|}{ Simpson grade } \\
\hline I & 280 & 29.8 \\
\hline$\|$ & 446 & 47.5 \\
\hline III & 103 & 11.0 \\
\hline IV & 106 & 11.3 \\
\hline V & 4 & 0.4 \\
\hline Simpson grade I/II & 726 & 77.3 \\
\hline Simpson grade III-V & 213 & 22.7 \\
\hline Simpson grade I-III & 829 & 88.3 \\
\hline Simpson grade IV/V & 110 & 11.7 \\
\hline \multicolumn{3}{|l|}{ Recurrence } \\
\hline Yes & 112 & 11.9 \\
\hline No & 827 & 88.1 \\
\hline \multicolumn{3}{|l|}{ Adjuvant radiation } \\
\hline Yes & 88 & 12.2 \\
\hline No & 631 & 87.8 \\
\hline
\end{tabular}

tion, residual tumor volume, WHO grade, and Simpson grade, high-grade histology (HR 3.40, 95\% CI 1.78-6.49; $\mathrm{p}<0.001$ ) and postoperative tumor volume (HR 1.05, 95\% CI 1.02-1.08; $\mathrm{p}<0.001)$ but not Simpson grade ( $\mathrm{p}=$ 0.398 ) were identified as predictors for recurrence (Table 2). Similar results were found when including the dichotomized EOR into the multivariate models. Hence, the risk of recurrence was similar after Simpson grade $\geq$ III (HR $1.20,95 \%$ CI $0.58-2.45 ; \mathrm{p}=0.624)$ or Simpson grade $\geq$ IV (HR 1.03, 95\% CI 0.42-2.56; $\mathrm{p}=0.945$ ) resections (for reference see Tables 1 and 2), while associations between tumor recurrence and residual tumor volume remained unchanged.

\section{Cutoff Threshold of Postoperative Residual Tumor Volume}

The regression tree model revealed a predictive cutoff value of tumor remnants of $>0 \mathrm{~cm}^{3}$ for prediction of shorter PFI. Moreover, risk of recurrence exponentially increased with increasing postoperative tumor volume (Fig. 3).

\section{Discussion}

After its initial description in $1957,{ }^{2}$ numerous studies 

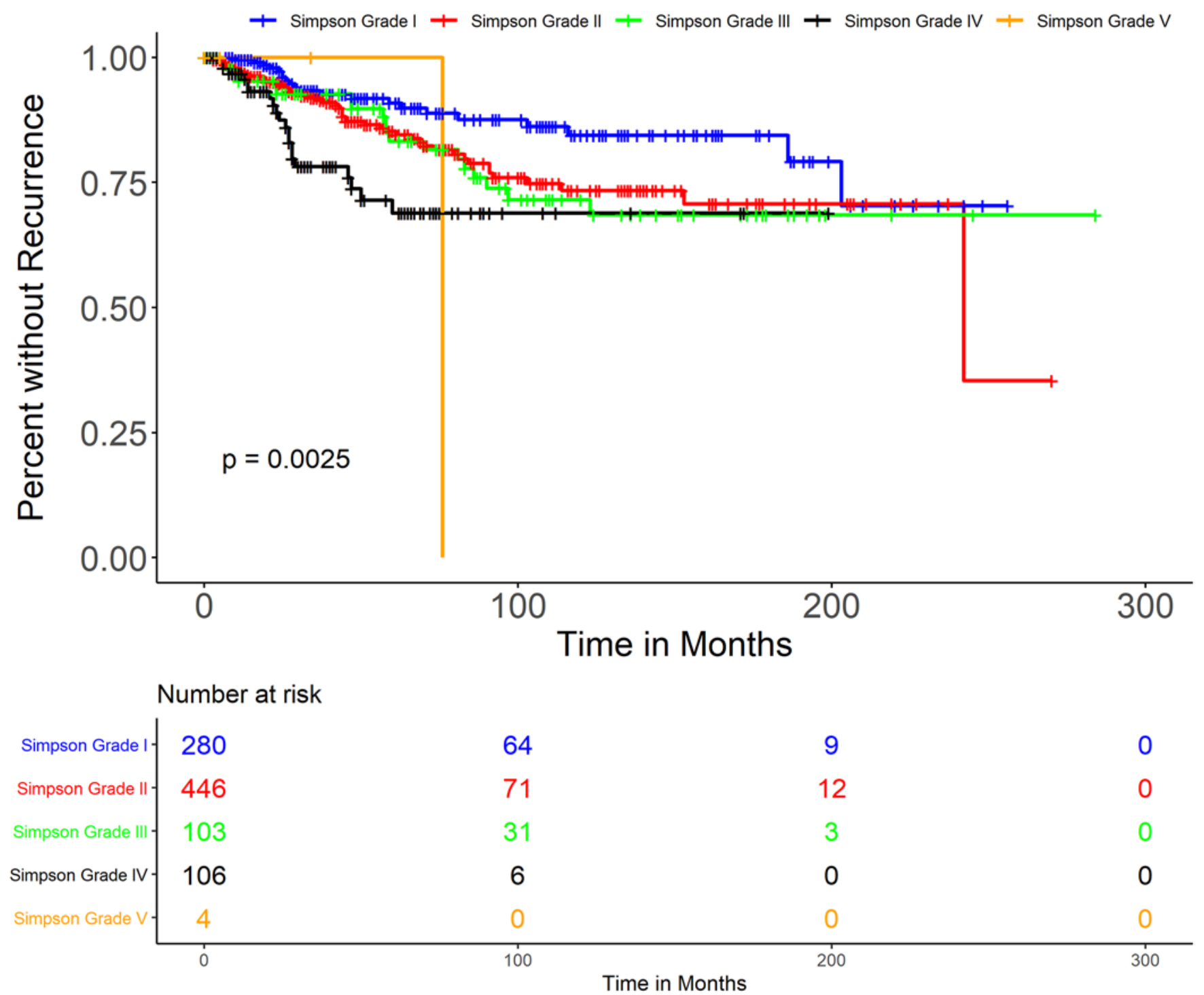

FIG. 2. Kaplan-Meier plots of the PFI after Simpson grade I-V resections. PFI strongly correlated with recurrence $(p=0.003)$. Figure is available in color online only.

reported correlations of the EOR in terms of the Simpson grade with risk of tumor recurrence after meningioma surgery. ${ }^{5-18,20}$ Similar to these findings, in our series Simpson grading correlated well with both the rate of tumor recurrence and the PFI. While the PFI was similar after Simpson grade II and III resections, recurrence rates tended to be higher after grade III than after grade II resections $(18 \%$ vs $11 \%, p=0.070)$. Although from a biological perspective, a Simpson grade II resection appears to be more radical than a grade III resection, characteristics of the bipolar coagulation (e.g., duration, wattage, area, etc.) are not generally defined and were rarely described in detail in the operative reports. Conversely, there is an ongoing discussion about whether Simpson grade III resections should be classified as GTR or STR in dichotomized scales. Hence, this discussion might indicate the difficulty of a correct designation of a grade III resection and/or the borderline prognostic role of the bipolar coagulation. On the other hand, the value of postoperative MRI to distinguish Simpson grade II and III resections is limited. As expected, skull base tumor location was associated with both STR and an increased postoperative tumor volume as compared to non-skull base meningioma location.

TABLE 4. Volume of tumor remnants following resection of tumors with different Simpson grades

\begin{tabular}{ccccc}
\hline \multirow{2}{*}{$\begin{array}{c}\text { Simpson } \\
\text { Grade }\end{array}$} & No. of & \multicolumn{3}{c}{ Residual Tumor Volume } \\
\cline { 3 - 5 } & Patients & Min $\left(\mathrm{cm}^{3}\right)$ & $\operatorname{Max}\left(\mathrm{cm}^{3}\right)$ & Median $\left(\mathrm{cm}^{3}\right)$ \\
\hline I & 95 & 0.00 & 4.07 & 0.00 \\
\hline II & 235 & 0.00 & 33.50 & 0.00 \\
\hline III & 26 & 0.00 & 9.55 & 0.00 \\
\hline IVIV & 67 & 0.00 & 78.50 & 4.79 \\
\hline
\end{tabular}




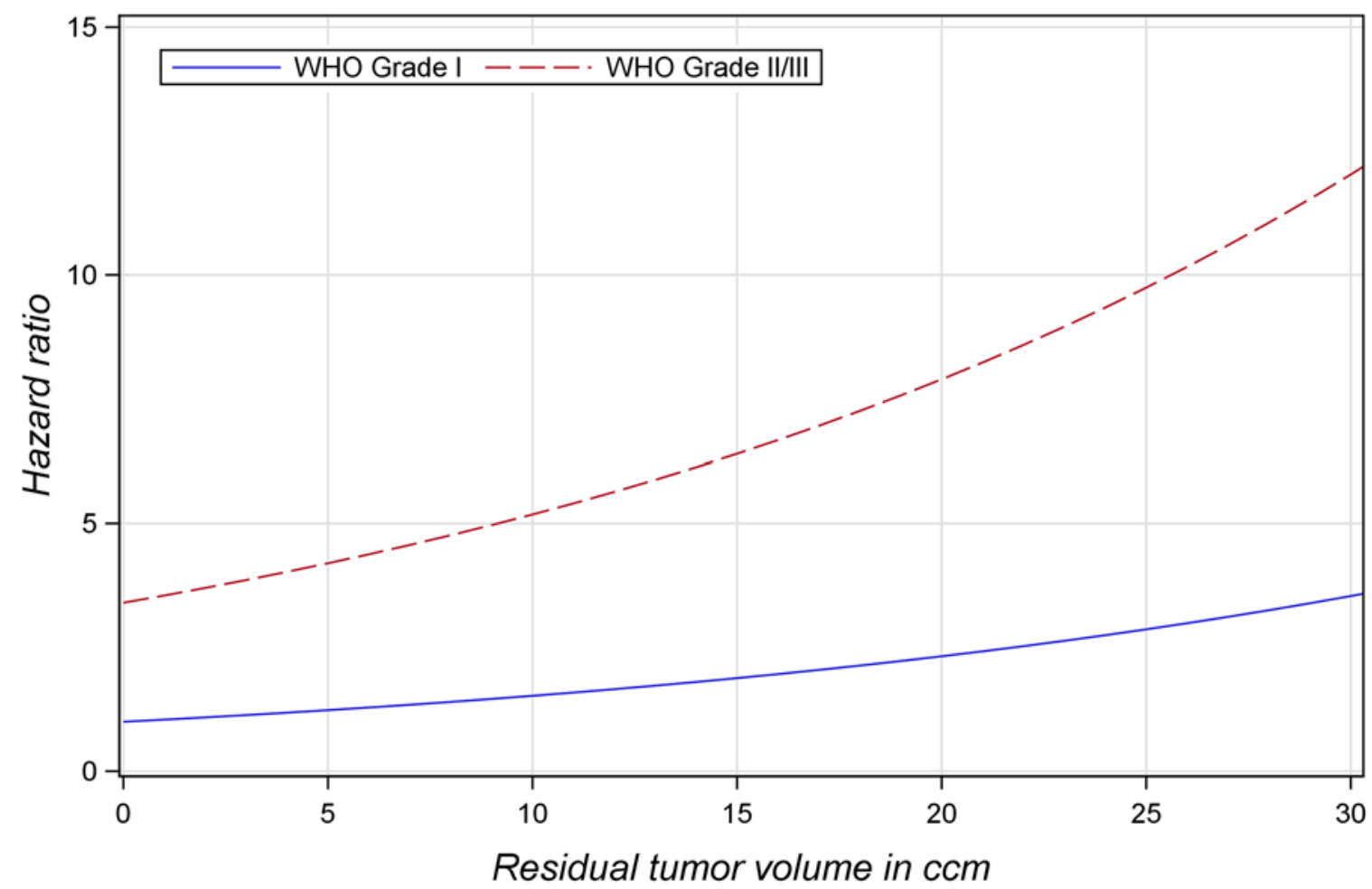

FIG. 3. Correlation between the volume of postoperative tumor remnants and the risk of recurrence. Remarkably, risk of recurrence (in terms of $\mathrm{HR}$ ) began to increase in the case of any detectable tumor remnant $\left(>0 \mathrm{~cm}^{3}\right)$ and exponentially increased with increasing residual tumor burden (reference: $0 \mathrm{~cm}^{3}$ tumor volume). Figure is available in color online only.

\section{Postoperative Imaging and Important Information About Tumor Remnants}

Despite remarkable advantages such as fast, easy, and imaging-independent assessment, intraoperative determination of the Simpson grade might suffer from subjectivity of the attending neurosurgeon. Correspondingly, a less favorable EOR on postoperative MRI as compared to the assessed Simpson grade was found in $20 \%$ in a recent series of 41 meningioma surgeries. In the same series, the authors revealed an intraclass correlation coefficient of 0.613 and an absolute agreement of $76 \%$ between the tumor volume on postoperative imaging and the intraoperatively assessed Simpson grade, and proposed a new radiological scale to quantify the EOR based on postoperative MRI (MEGA grading system). ${ }^{34}$ In our study, despite a sufficient reliability according to Cohen's kappa value, residual tumor tissue of up to $33.5 \mathrm{~cm}^{3}$ was detected in $8 \%$ of surgeries in which visible tumor tissue had been totally removed according to the intraoperative impression of the neurosurgeon (Simpson grade I-III resections). These findings indicate that correct intraoperative assessment might be complicated, such as in cases of limited overview of the entire resection cavity, and can lead to substantial misjudgment of the EOR. Further investigation of this subgroup revealed low residual tumor volumes in general, as well as skull base location or infiltration of the venous sinus in most of the cases. In fact, the patient with residual tumor volume of $33.5 \mathrm{~cm}^{3}$ and Simpson grade II resection harbored a meningioma infiltrating the superior sagittal sinus. However, the first postoperative imaging was performed 3 months after surgery, so that tu- mor regrowth appears to be unlikely. Aside from incorrect information of the patient and eventually wrong estimation of the risk of recurrence, tumor remnants after intraoperative inadvertently diagnosed GTR can lead to a delay or even failure of administration of adjuvant radiation (e.g., in atypical meningiomas) ${ }^{1}$ and might also impact study inclusion. Moreover, our results also support the hypothesis that intraoperative MRI might be helpful to detect residual tumor tissue in selected cases, such as when GTR of meningiomas in anatomical locations with limited intraoperative overview is intended. Interestingly, remnants were mostly larger and more commonly observed after Simpson grade III than grade II resections, eventually indicating secondary tumor shrinkage after bipolar coagulation.

In contrast, Simpson grade IV and V resections potentially subsume an arbitrary amount of residual tumor tissue and can therefore only approximately quantify the extent of tumor removal. Correspondingly, postoperative tumor volume in our study ranged from 0.00 to $78.5 \mathrm{~cm}^{3}$ after Simpson grade V resections. The absence of detectable tumor remnants on postoperative MRI following Simpson grade IV resection might additionally reflect the difficulty of assessment of the Simpson grade in some cases and further highlights advantages of postoperative radiological imaging.

\section{Prognostic Value of the Postoperative Tumor Volume Compared to Intraoperatively Assessed EOR}

While it appears reasonable that the amount of postoperative vital tumor tissue impacts the risk of relapse, associa- 
tions are sparsely investigated, and volumetric analyses are mostly restricted to meningioma subgroups and small patient groups. ${ }^{23,34-37}$ In 2018, Hunter et al. ${ }^{23}$ reported a strong correlation between the postoperative tumor volume and recurrence in a series of 23 petroclival meningiomas. Similarly, Shakir et al. ${ }^{35}$ showed that local control is strongly correlated with the postoperative tumor volume prior to adjuvant radiation in a series of 70 atypical meningiomas and delineated a critical cutoff value of approximately $9 \mathrm{~cm}^{3}$. Fujimoto et al., ${ }^{38}$ however, outlined residual tumor volume of $\geq 3 \mathrm{~cm}^{3}$ as a predictor of progression in uni- and multivariate analysis. In contrast, our group could not determine a prognostic impact of the postoperative tumor volume in 49 subtotally (Simpson grade $\geq$ IV) resected skull base meningiomas in a previous study. ${ }^{21}$ Likewise, Materi et al. could not identify residual tumor volume as a predictor of recurrence in multivariate analysis in 141 subtotally resected WHO grade I meningiomas. However, residual tumor volume was correlated with a high absolute annual growth rate with a significant threshold value of $5 \mathrm{~cm}^{3} .{ }^{37}$ In the current series of meningiomas of all intracranial locations, we demonstrated a strong correlation between recurrence and the postoperative tumor volume. It was noteworthy that the risk of recurrence increased exponentially with a critical cutoff value of $0 \mathrm{~cm}^{3}$, indicating that one should strive for maximum achievable tumor resection whenever safely feasible. Moreover, the prognostic value of the postoperative tumor volume was distinctly superior as compared to the intraoperatively assessed EOR, including the Simpson grade and both established dichotomized scales. To the best of our knowledge, no previous studies exist that directly compare the predictive value of surgical and radiological EOR. While the informative gain of early postoperative imaging after meningioma surgery remains to be further elucidated, ${ }^{22,34}$ these findings suggest that tumor volumetry on the initial postoperative MRI should be additionally considered when estimating the risk of recurrence in patients with meningioma. In Fig. 3, we provide a useful tool to estimate the risk of recurrence by the volume of postoperative tumor remnants in clinical routine. In view of our results, the question also arises as to whether the Simpson classification system currently remains the most appropriate method to determine the EOR in meningiomas. However, the Simpson classification system carries important advantages such as easy intraoperative assessment independent of the availability of imaging, as well as information about the coagulation of the dural attachment, which is not presented by postoperative MRI. Thus, measurement of the residual tumor volume should be considered a useful adjunct to the Simpson classification system to predict the risk of postoperative tumor recurrence.

\section{Limitations of the Study}

We are aware of some limitations of the study. Information about adjuvant radiation could not be obtained in detail and was therefore not factored into the uni- and multivariate analyses. Because routine early postoperative imaging was typically not performed, we considered the first postoperative MRI within 6 months after surgery for volumetry and subsequent analyses. Hence, we cannot exclude the possibility that early tumor progression eventu- ally partially contributed to postoperative tumor volume, which might explain discrepancies between the intraoperative assessment of the EOR and the postoperative tumor volume in some cases. In addition, imaging was performed in our hospital as well as in outpatient radiological departments, leading to a heterogeneous quality in terms of slice thickness and triplanar reconstructions. Although imaging was critically analyzed by a team of two independent observers, and preoperative scans as well as operative reports were considered, tumor tissue within the venous sinus and postoperative reactive changes (such as scar formation, etc.) might have complicated exact postoperative volumetry, and controversial cases were ultimately judged by discussion. Nevertheless, disclosure of interrater variability is missing. According to the standard treatment and clinical decision-making, tumor progression was diagnosed in cases of any detected tumor growth, but not according to recently proposed Response Assessment in Neurooncology (RANO) criteria. ${ }^{36}$ Despite the potential collinearity between postoperative tumor volume and the EOR, we included both variables in multivariate analysis, eliminating the less significant parameter by backward stepwise logistic regression. Finally, although this study provided investigations in a large cohort with sufficient follow-up information, it suffers from its retrospective nature.

\section{Conclusions}

Our findings strongly emphasize the informative gain of the initial postoperative MRI after meningioma surgery. Imaging reveals tumor remnants in a considerable portion of patients. The postoperative tumor volume predicts the risk of recurrence more relevantly than the Simpson grade and both derived, established, dichotomized scales. Hence, postoperative imaging should be carefully considered when estimating the risk of postoperative tumor recurrence. The question arises as to whether early postoperative imaging after meningioma surgery should be established to improve prediction of prognosis and/or planning of adjuvant treatment or study inclusion.

\section{References}

1. Goldbrunner R, Minniti G, Preusser M, et al. EANO guidelines for the diagnosis and treatment of meningiomas. Lancet Oncol. 2016;17(9):e383-e391.

2. Simpson D. The recurrence of intracranial meningiomas after surgical treatment. J Neurol Neurosurg Psychiatry. 1957; 20(1):22-39.

3. Jenkinson MD, Javadpour M, Haylock BJ, et al. The ROAM/ EORTC-1308 trial: Radiation versus Observation following surgical resection of Atypical Meningioma: study protocol for a randomised controlled trial. Trials. 2015;16:519.

4. Jenkinson MD, Santarius T, Zadeh G, Aldape KD. Atypical meningioma-is it time to standardize surgical sampling techniques? Neuro Oncol. 2017;19(3):453-454.

5. Adegbite AB, Khan MI, Paine KW, Tan LK. The recurrence of intracranial meningiomas after surgical treatment. $\mathrm{J} \mathrm{Neu}$ rosurg. 1983;58(1):51-56.

6. Bumrungrachpukdee P, Pruphetkaew N, Phukaoloun M, Pheunpathom N. Recurrence of intracranial meningioma after surgery: analysis of influencing factors and outcome. $J$ Med Assoc Thai. 2014;97(4):399-406.

7. Cao X, Hao S, Wu Z, et al. Survival rates, prognostic factors 
and treatment of anaplastic meningiomas. J Clin Neurosci. 2015;22(5):828-833.

8. Durand A, Labrousse F, Jouvet A, et al. WHO grade II and III meningiomas: a study of prognostic factors. J Neurooncol. 2009;95(3):367-375

9. Gallagher MJ, Jenkinson MD, Brodbelt AR, et al. WHO grade 1 meningioma recurrence: Are location and Simpson grade still relevant? Clin Neurol Neurosurg. 2016;141:117-121.

10. Gousias K, Schramm J, Simon M. The Simpson grading revisited: aggressive surgery and its place in modern meningioma management. J Neurosurg. 2016;125(3):551-560.

11. Hasseleid BF, Meling TR, Rønning P, et al. Surgery for convexity meningioma: Simpson Grade I resection as the goal: clinical article. J Neurosurg. 2012;117(6):999-1006.

12. Heald JB, Carroll TA, Mair RJ. Simpson grade: an opportunity to reassess the need for complete resection of meningiomas. Acta Neurochir (Wien). 2014;156(2):383-388.

13. Nanda A, Bir SC, Maiti TK, et al. Relevance of Simpson grading system and recurrence-free survival after surgery for World Health Organization Grade I meningioma. J Neurosurg. 2017;126(1):201-211.

14. Nanda A, Bir SC, Konar S, et al. World Health Organization Grade I convexity meningiomas: study on outcomes, complications and recurrence rates. World Neurosurg. 2016;89: 620-627.e2.

15. Oya S, Kawai K, Nakatomi H, Saito N. Significance of Simpson grading system in modern meningioma surgery: integration of the grade with MIB-1 labeling index as a key to predict the recurrence of WHO Grade I meningiomas. $J$ Neurosurg. 2012;117(1):121-128.

16. Pettersson-Segerlind J, Orrego A, Lönn S, Mathiesen T. Long-term 25-year follow-up of surgically treated parasagittal meningiomas. World Neurosurg. 2011;76(6):564-571.

17. Sughrue ME, Kane AJ, Shangari G, et al. The relevance of Simpson Grade I and II resection in modern neurosurgical treatment of World Health Organization Grade I meningiomas. J Neurosurg. 2010;113(5):1029-1035.

18. Yamaguchi S, Terasaka S, Kobayashi H, et al. Prognostic factors for survival in patients with high-grade meningioma and recurrence-risk stratification for application of radiotherapy. PLoS One. 2014;9(5):e97108.

19. Schipmann S, Schwake M, Sporns PB, et al. Is the Simpson grading system applicable to estimate the risk of tumor progression after microsurgery for recurrent intracranial meningioma? World Neurosurg. 2018;119:e589-e597.

20. Voß KM, Spille DC, Sauerland C, et al. The Simpson grading in meningioma surgery: does the tumor location influence the prognostic value? J Neurooncol. 2017;133(3):641-651.

21. Brokinkel B, Stummer W, Sporns P. Simpson grade IV resections of skull base meningiomas: does the postoperative tumor volume impact progression? J Neurooncol. 2018;137(1): 219-221.

22. Geßler F, Dützmann S, Quick J, et al. Is postoperative imaging mandatory after meningioma removal? Results of a prospective study. PLoS One. 2015;10(4):e0124534.

23. Hunter JB, O'Connell BP, Carlson ML, et al. Tumor progression following petroclival meningioma subtotal resection: a volumetric study. Oper Neurosurg (Hagerstown). 2018;14(3): $215-223$.

24. Gaonkar B, Macyszyn L, Bilello M, et al. Automated tumor volumetry using computer-aided image segmentation. Acad Radiol. 2015;22(5):653-661.

25. Laukamp KR, Thiele F, Shakirin G, et al. Fully automated detection and segmentation of meningiomas using deep learning on routine multiparametric MRI. Eur Radiol. 2019; 29(1):124-132.

26. Xie K, Yang J, Zhang ZG, Zhu YM. Semi-automated brain tumor and edema segmentation using MRI. Eur J Radiol. 2005;56(1):12-19.
27. Adeli A, Hess K, Mawrin C, et al. Prediction of brain invasion in patients with meningiomas using preoperative magnetic resonance imaging. Oncotarget. 2018;9(89):3597435982.

28. Brokinkel B, Holling M, Spille DC, et al. Surgery for meningioma in the elderly and long-term survival: comparison with an age- and sex-matched general population and with younger patients. J Neurosurg. 2017;126(4):1201-1211.

29. Hess K, Spille DC, Adeli A, et al. Brain invasion and the risk of seizures in patients with meningioma. J Neurosurg. 2018; 130(3):789-796.

30. Sicking J, Voß KM, Spille DC, et al. The evolution of cranial meningioma surgery-a single-center 25-year experience. Acta Neurochir (Wien). 2018;160(9):1801-1812.

31. Spille DC, Heß K, Sauerland C, et al. Brain invasion in meningiomas: incidence and correlations with clinical variables and prognosis. World Neurosurg. 2016;93:346-354.

32. Péus D, Newcomb N, Hofer S. Appraisal of the Karnofsky Performance Status and proposal of a simple algorithmic system for its evaluation. BMC Med Inform Decis Mak. 2013; $13: 72$.

33. Louis DN, Ohgaki H, Wiestler OD, Cavenee WK, eds. WHO Classification of Tumours of the Central Nervous System. Revised 4th edition. International Agency for Research on Cancer; 2016.

34. Slot KM, Verbaan D, Bosscher L, et al. Agreement between extent of meningioma resection based on surgical Simpson grade and based on postoperative magnetic resonance imaging findings. World Neurosurg. 2018;111:e856-e862.

35. Shakir SI, Souhami L, Petrecca K, et al. Prognostic factors for progression in atypical meningioma. J Neurosurg. 2018; 129(5):1240-1248.

36. Huang RY, Unadkat P, Bi WL, et al. Response assessment of meningioma: $1 \mathrm{D}, 2 \mathrm{D}$, and volumetric criteria for treatment response and tumor progression. Neuro Oncol. 2019;21(2): 234-241.

37. Materi J, Mampre D, Ehresman J, et al. Predictors of recurrence and high growth rate of residual meningiomas after subtotal resection. J Neurosurg. Published online January 3, 2020. doi:10.3171/2019.10.JNS192466

38. Fujimoto T, Ishida Y, Uchiyama Y, et al. Radiological predictive factors for regrowth of residual benign meningiomas. Neurol Med Chir (Tokyo). 2011;51(6):415-422.

\section{Disclosures}

The authors report no conflict of interest concerning the materials or methods used in this study or the findings specified in this paper.

\section{Author Contributions}

Conception and design: Spille, B Brokinkel. Acquisition of data: Spille, Hess, C Brokinkel, B Brokinkel. Analysis and interpretation of data: Spille, Paulus, Stummer, B Brokinkel. Drafting the article: Spille, B Brokinkel. Critically revising the article: Hess, Warneke, Mawrin, Paulus, B Brokinkel. Reviewed submitted version of manuscript: all authors. Approved the final version of the manuscript on behalf of all authors: Spille. Statistical analysis: Bormann, Sauerland, Stummer. Study supervision: Paulus, Stummer, B Brokinkel.

\section{Correspondence}

Dorothee Cäcilia Spille: University Hospital Münster, Germany. dorotheecaecilia.spille@ukmuenster.de. 